\title{
ESPECIAL INCIDENCIA DEL ACTUAL REGIMEN DE LA FUNCION PUBLICA ESPAÑOLA EN EL ESTADO, COMUNIDADES AUTONOMAS Y ADMINISTRACION LOCAL
}

por

\section{Carlos Carrasco Canals}

SUMARIO: I. INTRODUCCION.-II. LA FUNCION PUBLICA DEL ESTADO.-III. LA FUNCION PUBLICA DE LAS COMUNIDADES AUTONOMAS.-IV. LA FUNCION PUBLICA LOCAL: 1. UNA REFORMA CON DOS ORDENAMIENTOS: LA LEY DE MEDIDAS PARA LA REFORMA DE LA FUNCIÓN PÚBLICA Y LA LEY REGULADORA DE LAS BASES DEL REGIMEN LOCAL. 2. EL ESTATUTO DEL FUNCIONARIO LOCAL. 3. LA APLICACION DIRECTA, SUBSIDIARIA, SUPLETORIA O POR VÍA DE REENVÍO DE LA LEY DE MEDIDAS EN LA Vida local. 4. PROBlemas aCTUales de la FunCión PÚbliCa LOCAL EN ESPAÑA. 5. INTENTO DE UNA SÍNTESIS A TRAVÉS DE DOS ESTATUTOS DE FUNCIONARIOS: EL ESTATUTO GENERAL DE LA FUNCIÓN PÚBLICA Y EL ESTATUTO PARTICULAR DE LA LOCAL.-V. LA REFORMA BUROCRATICA ESPAÑOLA, ACONTECIMIENTO IMPORTANTE.

\section{INTRODUCCION}

Recientemente nos hemos ocupado de la función pública en España, al examinar la reforma y sus perspectivas de futuro (1).

(1) Vid. Carrasco Canals, C.: "La Función Pública española en la actualidad (la reforma y perspectivas de futuro)", Revista de Administración Pública. Centro de Estudios Constitucionales, num. 107, mayo-agosto 1985, pp. 259-327, en que se estudia la Ley de Medidas para la Reforma de la Función Pública en España, desde los más diversos aspectos. 
Asistimos también, en el marco de la integración de España con las Comunidades Europeas a las «Premiers rencontres francoespagnoles sur les régimes de fonction publique», celebradas en París (30 de septiembre a 1 de octubre de 1985) (2).

Todo ello es motivo para encarar de nuevo este tema, de alguna manera huérfano de tratamiento doctrinal (3).

Este trabajo es de reflexión, sin vocación dogmática, supone una

(2) En el marco de la integración de España con la Comunidad Europea y el incremento de las relaciones cientificas franco-españolas que resultará necesario. la UER de Ciencias Junidicas de la Universidad de Paris X (Nanterre). organizo unas Jornadas de estudio comparado. alternativo entre Francia y Espana. sobre las Ädministraciones de ambos paises y en especial sobre sus regimenes de funcion publica. proximos en muchos aspectos. pero caracterizados tambien por naturales especificidades.

Con el patronato conjunto del Instituto Internacional de Administracion Publica (IIAP). del Instituto Francès de Ciencias Administrativas (IFSA) y de la UER de Ciencias Juridicas de la Universidad de Paris X. se celebro la primera de las reuniones. durante los dias 30 de septiembre y 1 de octubre de 1985. en la sede del IIAP. 2. A venue de l'Observatoire. Paris.

Las ponencias que se presentaron fueron las siguientes:

1.a "Estatuto de la Función Pública del Estado". por M. P. Gaborit. Profesor de Ciencias Politicas y Director General de la Juventud (Ministerio de la Juventud y el Deporte), y por D. Juan-Alfonso Santamaria Pastor. Catedratico de Derecho Administrativo de Universidad.

2.a "La Función Pública Territorial», por M. O. Schrameck, Letrado del Consejo de Estado (Maitre des Requetes) y Director General de Enseñanza Superior, y por D. Santiago MuNoz MaChado, Catedrático de Derecho Administrativo en la Universidad de Alcalá de Henares.

3.a "La Formación de Funcionarios", por Mme. S. Trosa, Consejero Ténico en el Ministerio del Interior y de la Descentralización, y por D. Luciano PAREJo ALFonso. Subsecretario del Ministerio de Administracion Territorial y Catedrático de Derecho Administrativo.

4. "Politización y Alternancia Politica», por M. J. Costa, Letrado del Consejo de Estado y Secretario General del Instituto Francés de Ciencias Administrativas, conjuntamente con M. H. MaIsL, Profesor de Derecho Püblico en la Universidad de Paris X (Nanterre), y por D. a Amparo Rubiales, Secretaria General de Relaciones con el Parlamento Andaluz, de la Junta de Andalucia, y Profesora de Derecho Administrativo.

5. a Mesa Redonda sobre la "Función Pública Hispano-Francesa". Intervinieron en esta Mesa Redonda los siguientes señores:

Representacion francesa: M. J. LE GARREC. Secretario de Estado Encargado de la Función Pública y de la Simplificación Administrativa. M. E. GıUILY, Director General de Administración Local. M. P. PERRIN, Secretario General de la Comunidad Urbana de Lille. M. J. P. Puissochet, Director del Instituto Internacional de Administración Pública.

Representación española: D. Jaime MONTALVo, Director del Instituto Nacional de Administración Pública (INAP) y Catedrático de Derecho del Trabajo de la Universidad. D. Carlos Carrasco Canals, Director de la Escuela Nacional de Administración Local (ENAL) del Instituto de Estudios de Administracion Local y Profesor Titular de Derecho Administrativo de la Universidad.

(3) Tal vez por lo reciente del ordenamiento, tambien por razones obvias de implementación de una ambiciosa reforma, por el caracter de la misma, no disponemos hasta la fecha de aportaciones doctrinales; excepciones significativas y parciales las hay, como el discurso leido por GARRIDO FALLA, Fernando, el dia 19 de febrero de 1985, para su ingreso como Académico de Numero de la Real Academia de Ciencias Morales y Politicas, sobre: "Reformas de la Función Pública", 106 paginas en cuarto, Madrid, 1985. Cfr. también la contestación del Academico LOPEZ RODO, Laureano, incluida en el mismo folleto, que publica la Academia de referencia. 
aportación por aproximación, donde a manera de catálogo se enumeran cuestiones, se sugieren alternativas, por inducción se producen interrogantes y por supuesto es intento de mostrar la rica variedad producida por la reforma de la función pública en España. Reforma burocrática, que afecta de forma directa y sensible a la Administración Local (4).

Existen grandes temas, como el de la existencia o no de un estatuto de la función pública, realidades en nuestro país, como la complejidad de la función pública territorial, aspectos técnicos de la formación funcionarial y, por último, incidencia de la politización o de la alternancia política en la función pública.

En cuanto al estatuto de la función pública, la primera cuestión que se plantea es si debe existir uno solo, el del Estado; si varios, según las Administraciones públicas, o si bien es necesario determinar un estatuto general para todos los funcionarios, con especificaciones por colectivos, que no necesariamente Cuerpos.

También esta situación debe ser contemplada desde las más diversas perspectivas y úpticas, la rigidez, la complejidad y la posible politización estatutaria, serían aspectos notables en este ámbito.

También se puede afrontar esta temática problemática, desde el punto de vista del administrado, no solamente del administrador, que es lo usual.

La idea estatutaria es un concepto preconcebido, convencional, que atraviesa diversos momentos, que tiene distintas declaraciones de intenciones, que sectorializa intereses, con un desarrollo distinto, que se llega a ella desde una perspectiva legal o, en su caso, jurisprudencial, a través de una práctica judicial, que resuelve en conflicto las situaciones funcionariales.

Otra cuestión es la de la politización de los temas relativos a la función pública, por eso se habla tradicionalmente de politización o de alternancia política, e influencia de esta alternancia en las Administraciones públicas.

(4) Este ha sido puesto de relieve recientemente por Gonzalez-Haba GuiSado, Vicente Maria, en la RAP núm. 107, mayo-agosto de 1985, pp. 329-356, en un trabajo titulado "La función pública local en el marco de la reforma burocrática española", si bien se refiere no al texto de la Ley Reguladora de las Bases del Régimen Local, sino al Proyecto, por razón del tiempo en que se redactó el citado articulo de revista.

Tambièn en Revista de Estudios de la Vida Local (IEAL) num. 224, octubre-diciembre de 1984, año XLIII, vid. el articulo de BocANEGRA SIERRA, Raúl: "La función pública en el Proyecto de Ley Reguladora de Bases del Régimen Local», pp. 431-474. 
El reparto de la función pública entre el Estado, las Comunidades Autónomas y las Corporaciones locales diseñan un esquema que es distinto en cada país (5). Es típico el sistema francés hasta hace bien poco, donde el régimen de la función pública no es aplicable a las colectividades locales y existía una tradicional diferencia entre agentes y funcionarios, los servidores de las colectividades locales eran agentes y los del Estado funcionarios. Si bien, a partir de 1981 esta situación varía en el país vecino, no obstante, una multisecular tradición todavía incide y de alguna manera existen dos bloques normativos, a pesar de haber un estatuto general de funcionarios.

Temas como la codificación de las normas funcionariales, la modernización, el funcionamiento y el acceso a las tareas de la función pública, la transparencia en la gestión, la participación, la comunicación, son aspectos referidos al aparato funcionarial y de trascendencia.

Para examinar la función pública hay que delimitar su ámbito, sentido y contenido, perspectiva y consideración metodológica, dificultades en un momento coyuntural y coexistencia de los más diversos factores, como es el supuesto español, donde se produce un cambio y modernización pero existen elementos tradicionales de estabilidad.

Deslindar la función pública en sentido estricto, no incluir una posible función pública paralela, actividad burocrática de sindicatos o partidos, personal de confianza que realiza funciones burocráticas o con trascendencia en este sentido, no es una fácil tarea. Tampoco se puede incluir en este momento la función pública diferenciada o diversificada como puede ser la función desarrollada por la Administración militar que queda al margen en este momento de delimitación.

De otra parte; la perspectiva metodológica imprime también carácter; al hacer cualquier estudio sobre temas como el tratado, se puede examinar la cuestión jurídica, política y socialmente.

El supuesto español, superadas las dificultades intrínsecas del

(5) Sobre los medios personales de la Administración Local en España, vid. LLISET Borrel, Francisco: Manual de Derecho Local, Publicaciones Abella, El Consultor de los Ayuntamientos, Madrid,. 1985, p. 379 y ss.

Cfr. PAREJo Alfonso, Luciano: Estado social y Administración pública, los postulados constitucionales de la reforma administrativa. Editorial Civitas, Madrid, 1983, 326 páginas en octavo. 
cambio, es un caso interesante, pues responde a una situación, a unos supuestos de hecho, a un funcionariado, pero además en un contexto político, en una nueva estructura del Estado, el autonómico, $y$ en un entorno y marco de integración con las Comunidades Europeas.

Detrás de la reforma de la función pública existe una ideología, ideología política evidentemente, que es llevada a la práctica con una lógica peculiar, con impulsos y contenidos, que responden a unas ideas que subyacen, y fundamentalmente son el anticorporativismo, adecuación a las necesidades actuales y a un marco constitucional y diversos parámetros que han sido detectados, desde el coloquio a la prensa diaria y en conferencias, reuniones o foros, del más diverso talante.

La situación del actual régimen de la función pública española, por comparación con la de otros países próximos al nuestro, no dista ni en problematicidad ni en singularidad específica; de alguna manera nos encontramos en un régimen estatutario, como situación jurídica objetivizada.

Existen características particulares del funcionariado español, como cuerpo social conservador, poco móvil, con iniciativa escasa y con un grado mínimo de politización. El funcionario español está relativamente poco profesionalizado.

El panorama actual ha experimentado, con el nuevo Estado autonómico, un giro importante al reestructurarse la Administración territorial. Desaparece la centralización al uso napoleónico y aparecen las autonomías, con transferencias que no es momento de evaluar.

Existen fenómenos típicos en nuestra función pública, que convendría valorar, como es el de la composición por edades y el impacto producido por la anticipación de la jubilación a los 65 años, o por la aplicación de la Ley de Incompatibilidades.

Otro aspecto relevante, al que nos hemos de referir más adelante, es el de la función pública territorial, que nosotros llamamos incidencia del actual régimen sobre las Comunidades Autónomas constituidas en España.

Existen diversas opciones, dentro de la función pública territorial española y lógicamente un futuro próximo y una realidad en la actualidad.

Aspectos como la formación de los funcionarios, son de trascendencia y conllevan la consideración de diversos matices a los que 
nos hemos referido en ocasiones (6). La selección, la formación y el perfeccionamiento de los funcionarios, bajo el epígrafe genérico de "formación», es un problema y tema susceptible de resolver desde un punto de vista técnico. Conlleva problemas evidentes: financieros en primer lugar, de recursos y medios personales y materiales. Después se plantea una cuestión organizatoria, como es la creación de centros que se ocupen de estas tareas, su ubicación, naturaleza, organización y dependencia.

Es importante la consideración de aspectos que no han sido puestos de relieve lo suficientemente, sobre el derecho y el deber a la formación del funcionario, así como de la Administración. Esta es una cuestión previa que debe situarse en alternativa interrogante sobre si el funcionario tiene un derecho subjetivo a recibir una formación, requisito imprescindible para el desempeño de su función pública, o si este derecho le corresponde a la Administración pública de que se trate, o globalizada esta situación jurídica activa de derechos, éste corresponde al Estado. Congruentemente, podría plantearse la situación juridico-pasiva correspondiente de deberes, tanto del administrado cualificado que es el funcionario, como el de la Administración.

En el momento actual la situación, en relación con esta cuestión, se contempla en la Ley de Funcionarios de 1964, como un deber (7), pero se concibe como un deber condicionado $y$, en

(6) Vid. nuestro trabajo sobre: "Selección, formación y perfeccionamiento de funcionarios locales". Separata del libro Organización Territorial del Estado (Administración Local), vol. I. Instituto de Estudios Fiscales, en las Jornadas organizadas por la Direccion General de lo Contencioso del Estado en 1984 sobre "Organizacion Territorial del Estado" (pp. 711 a 724), Madrid, 1985.

(7) Vid. Parejo Alfonso, Luciano, en su Ponencia presentada en las "Premieres rencontres franco-espagnoles sur les regimes de fonction publique", celebradas en Paris en septiembre-octubre de 1985 , citadas anteriormente.

El articulo 33 del Decreto $315 / 1964$, de 7 de febrero, por el que se aprueba la Ley Articulada de Funcionarios Civiles del Estado, dice textualmente: "Los funcionarios de los Cuerpos generales tienen el deber de asistir, previa autorización del Subsecretario del Departamento en que presten sus servicios, a cursos de perfeccionamiento con la periodicidad y caracteristicas que establezca la Presidencia del Gobierno, sin perjuicio de las enseñanzas que se organicen en cada Ministerio en relación con la materia de su competencia" (el subrayado es nuestro). En este precepto se contempla una antinomia, como es el deber que parece se está preconizando, con la petición de autorización previa, lo que no tiene sentido, lo tendria si fuera relativa la autorización a cómo ha de cumplirse, o en que tiempo debe hacerse, pero no, como es el caso, que la autorización es genérica respecto del deber, de ahi la poca o escasa utilidad que ha tenido este precepto.

Como complemento, los articulos 34 y 35 de la Ley Articulada de Funcionarios de 1964 encomiendan al Centro de Formación y Perfeccionamiento de Funcionarios, después Escuela Nacional de Administración Pública y actualmente Instituto Nacional de Administración Pública, la organización de Cursos de Perfeccionamiento, indicando la trascendencia de los mismos, que se reduce a la anotación en la hoja de servicios de los interesados. 
realidad, con escasa trascendencia durante los veinte años de vigencia del precepto aludido.

Un planteamiento de correlato derecho-deber, parece ser el correcto y responde a la idea de posibilitar una promoción dentro de la carrera administrativa del funcionario.

Al existir un derecho del funcionario a la formación, este derecho debe conectarse con su posible promoción y debe protegerse dicho derecho para que no sea una simple declaración de intenciones, sino una realidad.

En el actual régimen de la función pública, al objetivar las normas para la selección del personal, se encarga a las Administraciones públicas para que cuiden especialmente la conexión entre las pruebás a superar y la adecuación a los puestos de trabajo que se hayan de desempeñar, arbitrando la fórmula de ejercicios prácticos para conseguir esta finalidad. La famosa crisis de las oposiciones, su escasa rentabilidad, quiere obviarse manteniéndola como sistema selectivo, pero adecuado para conseguir funcionarios debidamente capacitados.

También es característica del régimen introducido en la reforma por la Ley de Medidas, el fomento de la promoción interna, que se concibe como un mandato a las Administraciones públicas (8), pues es de su responsabilidad el facilitar.el ascenso desde Cuerpos o Escalas de grupo inferior, a otros superiores.

La Administración, como organización servicial regida por el principio de la eficacia, neutral en su actuación y necesitada de un mínimo de autonomía (9), como ha sido definida por la doctrina,

(8) Vid. el articulo 22 de la Ley 30/84, de 2 de agosto, de Medidas para la Reforma de la Función Pública, donde en el apartado 1 se hace este encargo de facilitar la promoción interna que consiste en el ascenso, estableciendose unos requisitos, que en su caso los determina el Ministerio de la Presidencia o-el órgano competente de las respectivas Administraciones públicas.

Este articulo, en el apartado aludido, es declarado básico del régimen estatutario por el artículo 1.3 de la propia Ley de Medidas.

(9) Sobre estas caracteristicas se ha insistido por la moderna doctrina administrativista; cfr. la $o b$. cit. de Luciano PAREJO ALFONSO sobre Estado social y Administración pública. El propio autor, en su Ponencia citada anteriormente sobre "El derecho y el deber a la formación del funcionario y de la Administración», manifestaba: " $1 .{ }^{\circ}$ La Administración es una organización servicial. 2. ${ }^{\circ}$ Regida por el principio de eficacia. 3. ${ }^{\circ}$ Neutral en su actuacion. 4. ${ }^{\circ}$ Necesitada de un minimo de autonomia".

La doctrina alemana, y especialmente FORSTHOFF antes que nadie, supo destacar que la Administración es la pieza capital en el cumplimiento del principio social propio del Estado actual que, además de ser un Estado de Derecho, es un Estado social. En virtud de esta finalidad debe afirmarse que no es la Administración el lugar donde debe resolverse el pluralismo politico; es decir, la Administración no se caracteriza por el principio repre- 
que insiste en la nota de servicio, pues la organización administrativa está al servicio del Estado, para la defensa de los intereses generales, se relaciona con la actividad de formación dirigida al funcionario o excitada por éste, para que la Administración se la facilite. Dicha formación debe dirigirse hacia determinadas ideas, fundamentalmente a que los funcionarios conozcan ese ambiente servicial, esa eficacia en la función, la neutralidad y también el nivel de autonomía en que puede desenvolverse el funcionario.

Las alusiones realizadas deben caracterizarse por cierto grado de iniciativa, es decir, que ser funcionario no significa una simple automación, respuesta a impulsos externos o internos, sino que debe exigirse al funcionario, en su caso permitírsele, y por supuesto fomentar esta iniciativa, en evitación de actitudes que, en su grado sumo, pueden comportar inhibición y se configuran estas actitudes, en el régimen disciplinario, como falta muy grave.

Son problemas técnicos y no existen respuestas indudables en si es el sistema educativo o la Administración la que debe proporcionar determinadas ideas, hábitos, conocimientos, capacidades, aunque se puede llegar a un compromiso de lo que corresponde al sistema educativo y lo que corresponde a la Administración, con tareas evidentes de formación, que deben deslindarse y ser un perfeccionamiento en el propio ejercicio de la función pública encomendada.

Por último, habrá que tener en cuenta temas trascendentales como los de la influencia política en la Administración, lo que se ha venido llamando politización; o la cuestión de que exista una Administración politizada y los factores que favorecen la politización de la función pública, entre ellos las transformaciones de los sistemas políticos, que tienden a una individualización del poder, así como el aumento del poder administrativo, donde los políticos dependen cada vez más de los Servicios, y por ello se rodean de

sentativo. (Para lograr la articulación de las alternativas politicas ya existen otras instituciones dentro del Estado. fundamentalmente las Cámaras parlamentarias.) La Administración se caracteriza por la nota de servicio; la organización administrativa está al servicio del Estado para la defensa de los intereses de la comunidad, pero de los que se "apropia» justamente a través del dato de la servicialidad.

$Y$ estos intereses deben ser obtenidos con eficacia. En el estricto ámbito interno administrativo, prima el principio estructural de la eficacia sobre cualquier otro, incluido el del pluralismo politico (éste se realiza en otros ámbitos, como ya he dicho). Y es que sucede que la legitimación de la Administración depende precisamente de la eficaz realización de los intereses generales a los que sirve; es una "legitimación teleologica». 
gabinetes de confianza, que son afines o partidarios políticos. De alguna manera, se da una politización administrativa y una administrativización de la política.

Se habla de alternancia política, en el sentido de que al existir un Gobierno y una oposición, cuando cambian los papeles, por procedimientos democráticos evidentemente, el Gobierno en el poder puede cambiar a funcionarios, incluso competentes, pero no leales o afines. Naturalmente, el cambio debe producirse con discreción, sin sectarismo, respondiendo a un principio deontológico, de que quien no sea capaz de emprender las reformas necesarias, debe ser removido, pero su situación no debe conducir al ostracismo, la venganza, el abandono o cualquier otra fórmula similar, tampoco que sea una recompensa su remoción, sino por el contrario, buscarle un acomodo y adecuación a sus condiciones, caracteres, disposición y preparación funcionarial.

Se debe procurar, en una situación de riesgo como es la de la politización en la Administración, actuar con ponderación y evitar desfases, disfunciones, acumulando experiencias, incorporando nuevas técnicas y nuevos derechos en la Administración y evitando que presidan los criterios políticos a una eficaz gestión administrativa cotidiana.

Iniciado el tema, exponemos a continuación y en síntesis una serie de conceptos, cada uno de ellos susceptible de tratamiento individualizado, pero por razones de brevedad en este momento sólo enunciados.

La reforma de la Función Pública española, por imperativo constitucional, tiene unas características singulares:

- Responde a un programa gubernamental.

- Es básica, desde una perspectiva legislativa.

- Supone un nuevo marco, derivado de la Constitución española.

- Imprescindible para adecuar las estructuras burocráticas y su funcionamiento a la Constitución.

- Reforma en profundidad.

- Modificación del sistema retributivo.

- Racionalización de la estructura, para acercar la Administración al ciudadano.

- Especificamente novedosa. 
- Intento de unificación del régimen jurídico de todas las Administraciones públicas.

Apartados, todos ellos, explicitados en la introducción, a manera de preámbulo, de la Ley de Medidas para la Reforma de la Función Pública.

\section{LA FUNCION PUBLICA DEL ESTADO}

En el ámbito de aplicación de la Ley 30/1984, de 2 de agosto, de Medidas para la Reforma de la Función Pública (desde ahora Ley de Medidas), se describe el proyecto de un auténtico Estatuto del Funcionario en España.

Afecta al personal encargado de la Función Pública del Estado, con los siguientes caracteres:

- Administración del Estado y de sus Organismos Autónomos.

- Personal civil al servicio de la Administración Militar y sus Organismos Autónomos.

- Personal funcionario de la Administración de la Seguridad Social.

Pueden dictarse normas especiales, por peculiaridades, al siguiente personal:

- Docente e investigador.

- Sanitario.

- De los Servicios Postales y de Telecomunicación.

- Personal destinado en el extranjero.

\section{LA FUNCION PUBLICA DE LAS COMUNIDADES AUTONOMAS}

Existe una nueva ordenación de la Función Pública, de las Comunidades Autónomas.

Hay una regulación de la situación de los funcionarios transferidos de otras Administraciones públicas.

Los Estatutos de las diecisiete Autonomías, actualmente existentes, ordenan mediante Ley de sus respectivas Asambleas Legislativas su propia Función Pública.

Las soluciones en los Estatutos de Autonomía son distintas, por 
lo general han asumido competencias en esta materia, con alguna excepción (10).

Agrupando las Comunidades Autónomas según las soluciones acordadas, en relación con la función pública, se producen las siguientes (11):

- Competencia exclusiva, respetando los derechos y obligaciones esenciales, que la legislación básica del Estado reconozca a los funcionarios públicos.

- Competencias para el desarrollo legislativo y régimen jurídico de sus funcionarios, de acuerdo con los criterios de la legislación estatal.

- Sometimiento a los principios y normas de organización del Estado a sus propios funcionarios.

- Establecimiento de principios para evitar duplicidades de cargos y funciones, así como la proliferación de la burocracia, con sometimiento al régimen, organización y funcionamiento de la Función Pública estatal (12).

- Competencias, dentro de los principios generales y normas básicas del Estado.

(10) La excepción se produce en el Principado de Asturias, pues en esta Comunidad Autónoma, al examinar su Estatuto, se observa que, respecto de los funcionarios, de la Función Pública en general, no se dice nada; sólo en relación con la organización y régimen juridico de la Administración autónoma, el articulo 32.2 manifiesta: "El Presidente del Principado de Asturias es el del Consejo de Gobierno, cuya actividad dirige, coordina la Administración de la Comunidad Autonoma, designa y separa a los consejeros y ostenta la suprema representación del Principado y la ordinaria del Estado de Asturias". Vid. Estatuto de Autonomia de Asturias. Instituto de Estudios de Administracion Local, Madrid. 1982, con un estudio preliminar y notas por Enrique ORDUÑA REBOLLO, en especial p. 8 y ss, en que se hace un análisis de la Junta General del Principado de Asturias y tampoco se alude a la Función Pública. No obstante, en la Disposición Transitoria Sexta, del mencionado Estatuto, se habla de los derechos adquiridos de los funcionarios transferidos; pero, en realidad, aunque es la única alusión no tiene significación de regularse dicha materia estatutariamente.

(11) Cfr. Carrasco Canals, Carlos: "La Función Publica española en la actualidad (la reforma y perspectivas de futuro)", articulo citado anteriormente, pp. 275 a 277 , donde se agrupan las Comunidades Autonomas en cinco apartados, que comprenden las 16 de las 17 Autonomias existentes, que han regulado este tema, según sus especiales caracteristicas, y que van desde la declaración de competencia exclusiva hasta una declaración de reenvio, que supone la regulación del tema por una Ley de la Asamblea correspondiente.

Cada uno de los grupos, y en su caso cada Autonomia, es citada con articulos y apartados de sus respectivos Estatutos, para identificar el tratamiento, matices y consideración de la ordenación de la Función Pública autonómica.

(12) Lo resaltamos por singular, la Comunidad Autonoma de Aragón, en el articulo 44.3 de su Estatuto dice textualmente: «En desarrollo del principio de economia y sin perjuicio de la eficacia, la Administración pública de la Comunidad Autonoma se organizara sobre la base de evitar la duplicidad de cargos o funciones y la proliferación de la burocracia" (el subrayado es nuestro). 
El problema, en la actualidad, es que no todas las Comunidades Autónomas tienen su Ley de Función Pública, no llegan a la mitad, y estas leyes están en distinta fase de elaboración, según las diferentes Autonomías.

\section{LA FUNCION PUBLICA LOCAL}

En la Función Pública Local se produce la reforma con especiales características, pues existen dos ordenamientos jurídicos: la Ley de Medidas y la Ley $7 / 1985$, de 2 de abril, Reguladora de las Bases del Régimen Local (desde ahora Ley de Bases).

Se propone un estudio sistemático, sintético, de las cuestiones, a nuestro juicio, más importantes que suponen la reforma de la Función Pública Local en España.

1. UNA REFORMA CON DOS ORDENAMIENTOS: LA LEY DE MEDIDAS PARA LA REFORMA DE LA FUNCIÓN PÚBLICA Y LA LEY REGULADORA DE LAS BASES DEL RÉGIMEN LOCAL

Este es el primer problema, la dualidad de regímenes jurídicos, en una cuestión problemática: la función pública local.

Los impactos normativos en un panorama y realidad muy sensible y compleja.

Por el número de afectados, por las organizaciones muy diversas y la multivariedad existente, es necesario un tratamiento diferenciado.

Hay que hacer unas precisiones:

a) Hacia un diseño de ambas normativas.

La Ley de Medidas es una Ley problemática:

- provisional

- básica, que no de bases

- modificación parcial

- reformadora

- derogatoria de un sistema

- régimen transitorio y adicional complejo

- estructura peculiar: breve.

La Ley de Bases es una Ley técnicamente más perfecta:

- autonomía local 
- básica del Municipio

- preámbulo descriptivo

- prelación de fuentes, nuevo planteamiento

- Título VIII: «estatuto»

- está impregnada de una nueva filosofía y con nuevas concepciones doctrinales

- contiene diversos mandatos y plazos de ejecución.

b) Analogías y diferencias entre ambos ordenamientos:

Analogías, ambas normas son del mismo talante:

- democráticas

- abordan el tema del artículo $149.1 .18 .^{\circ}$ de la Constitución española

- responden a un compromiso del Gobierno, en su programa electoral

Diferencias:

- estructuralmente distintas, mejor estructurada la Ley de Bases

- la Ley de Medidas breve, 33 artículos y varias disposiciones añadidas al articulado

- la Ley de Bases larga, 120 artículos y menos disposiciones añadidas

- una provisional y otra definitiva

- Ley de Bases, más claramente derogatoria

- ambas de bases, básicas pero no de bases para articular, ya están ambas articuladas, sin embargo distintas

- en la Ley de Bases no existe incrustación de dos textos, para refundir en uno, como ocurre en la Ley de Medidas

- una es Ley de Medidas, según su denominación, y la otra es de Bases

c) Relaciones entre ambos ordenamientos:

- la Ley de Medidas es aplicable al personal de todas las Administraciones públicas

- la Ley de Bases, en cuanto ordenamiento de la función pública, sólo se aplica al personal al servicio de las Entidades locales. 
2. El ESTATUTO DE FUNCIONARIO LOCAL

¿Qué es un estatuto?

¿Depende de la óptica y consideración para aplicarlo al ámbito local?

¿Tiene actualmente un estatuto el funcionario local?

En función de la primera interrogante se podría discernir sobre su naturaleza, características, singularidad, etcétera.

Las posibles soluciones se han de enmarcar en:

a) El ámbito jurídico normativo:

- el problema no es nuevo, ni tampoco baladí o intranscendente.

- acotar la aplicación no es tarea sencilla

- en principio, subjetivamente, parece simple la aplicación: estará circunscrita a los funcionarios locales.

- objetivando: las Corporaciones Locales, entes de cobertura de tercer grado, dentro del esquema Estado, Autonomías y Entes Locales.

Esto nos reconduce a temas capitales: relaciones interadministrativas, prelación fuente (art. $5 \mathrm{LB}$ ), etcétera.

Habrá que examinar el Título VII de la Ley de Bases y su incidencia en el régimen jurídico de la Función Pública Local.

b) Incidencias:

- la nueva estructura autonómica del Estado

- la nueva tipología: habilitación nacional, interdependencia de los funcionarios.

- los funcionarios de carrera "propios» de los Entes Locales y la posible intercambiabilidad entre Administraciones y Corporaciones Locales.

- la promoción interna, profesional, en sentido horizontal y vertical.

c) Problemática:

- lo evidente es la problematicidad de la cuestión; supone una respuesta cóngrua además de la carga que estas situaciones provocan; donde se ha de deslindar y todos los problemas de límites son de difícil solución. 

local.

La Ley de Bases supone una segunda Constitución para la Vida

El Estatuto de la Función Pública es un reto para el empleo público, en momentos de crisis, paro y desempleo.

\section{LA APLICACION DIRECTA, SUBSIDIARIA, SUPLETORIA O POR VÍA DE REENVIOO, DE LA LEY DE MEDIDAS EN LA VIDA LOCAL}

Es un problema importante de afectación. Aspectos como la dependencia orgánica, Ministerio de la Presidencia y de Economía y Hacienda, y su intervención en la Función Pública, la programación de efectivos, la oferta de empleo público, las transferencias de funcionarios, los Registros de personal, la objetivación en la selección, los sistemas de provisión de puestos de trabajo, la promoción y la formación profesional, las titulaciones exigidas, etcétera.

Pero estè panorama sigue complicándose, al obligar la Ley de Medidas a una racionalización de estructura de los Cuerpos y Escalas, a un sistema retributivo distinto, donde las retribuciones a nivel complementario establecen nuevos conceptos: nivel, complemento específico, complemento de productividad y gratificaciones; un nuevo régimen disciplinario y el importante tema de la Seguridad Social de los funcionarios locales.

La aplicación es directa en unos casos, en otros subsidiaria o supletoria y en algunos supuestos se produce un reenvío normativo.

\section{a) Aspectos cardinales de la Ley de Medidas}

Establece las bases del régimen estatutario de los funcionarios públicos.

La Ley de Medidas, en su artículo $1 .^{\circ}$, puntos 3, 4 y 5 , nos sitúa en el tema de las bases y determinación del ámbito de aplicación de la misma, en relación con la Administración Local, planteándose los siguientes problemas:

- bases del régimen estatutario de los funcionarios públicos.

- personal específico de la Administración del Estado, por alusiones legales

- interpretación de lo que la Ley de Bases entiende por tales. En este sentido el legislador ha seguido la doctrina y jurisprudencia constitucional del Tribunal correspondiente, que en Sentencia de 7 de abril de 1983, dice: «... por principios, 
bases y directrices hay que entender los criterios generales de regulación de un sector del ordenamiento jurídico o de una materia jurídica, que deben ser comunes a todo el Estado». Esto supone un sentido positivo: objetivos, fines y orientaciones, y en un sentido negativo representa un límite al ejercicio de las competencias exclusivas de las Comunidades Autónomas.

b) Los preceptos que, sin ser bases o básicos, son aplicables directamente

- Existen muchos artículos en la Ley de Medidas que no han sido declarados básicos y estatutarios, y pueden tener aplicación directa al sector local, a pesar de haber sido excluidos de este carácter.

- Como muestra de artículos que inciden directamente y, sin embargo, no tienen carácter básico, son todos aquéllos que hablan de la Administración en general o de las Administraciones públicas, y no están incluidos en el artículo 1.3, párrafo $2 .^{\circ}$, de la Ley de Medidas, apartado que declara básicos y afecta a más de la mitad del total articulado del texto.

- Como ejemplo, se pueden citar los artículo $2,3.2,4.2 a$ ) y 5 de la Ley de'Medidas.

\section{c) Carácter supletorio de la Ley de Medidas}

Según el artículo 1.5, la Ley de Medidas tiene carácter supletorio para todo el personal ai servicio del Estado y de las Administraciones públicas, no incluido en su ámbito de aplicación.

Con motivo de esta declaración hay una serie de rúbricas problemáticas, muy importantes y abundantes, sobre:

- Alcance y significado de la supletoriedad.

- ¿Cómo se resuelve la colisión entre Ley de Medidas y Ley de Bases?

- Prevalencia de ordenamientos que son promulgados en el tiempo, Ley de Medidas anterior y de Bases posterior. Por tanto, se pueden suscitar problemas de derogación, con regímenes distintos, vigentes y además muy recientes.

- Alcance de la hermenéutica jurídica, con incidencias "de integración normativa. 


\section{Problemas actuales de la Función pública local EN ESPAÑA}

Puede hacerse un elenco cuestionable sobre los distintos aspectos que son problemáticos: existencia de ordenamientos paralelos, relaciones interordinamentales, problemas orgánicos de los Entes Locales, que lo son de cobertura de tercer grado, incidiendo la autonomía local, financiamiento siempre insuficiente, con gran falta de uniformidad y disparidad evidente, existencia de organizaciones infra y supramunicipales, con el carácter, ambas, de Municipios, peculiaridades locales típicas, tradición de una Administración, en ocasiones esclerotizada, con usos, costumbres y prácticas que solucionan de manera muy distinta problemas diferentes o iguales, con disparidades evidentes que se deben unificar.

Otra cuestión importante es que dos Administraciones preeminentes, la del Estado y la de las Comunidades Autónomas respectivas, que inciden con evidencia en la Local, pero en el aspecto de funcionamiento, sobre todo en el supuesto del personal de las Administraciones Autonómicas, que en estos momentos se nutren de personal al servicio de la Administración Local. Se produce en la realidad, y hasta ahora, un vaciamiento de los cuadros de efectivos personales, en detrimento de la Administración Local.

Examen del personal de las Corporaciones Locales:

\section{a) El personal al servicio de las Entidades Locales:}

Así se titula el epígrafe del Título VII de la Ley de Bases, examinado más adelante.

En síntesis, actualmente la Función Pública Local se desenvuelve bajo tres grandes rúbricas, que califican al personal a su servicio:

- De carrera, es al único personal al servicio de la Administración Local que se denomina con la expresión tradicional de «funcionarios».

- Contratados en régimen de derecho laboral, se les llama, también, personal laboral de las Corporaciones Locales.

- Eventual, que desempeña puestos de confianza o asesoramiento especial, que es dintinto al personal de empleo eventual del régimen jurídico anterior.

- Desaparición de los contratados administrativos y resurrección en la Vida local de los interinos, que habian desaparecido, como figura funcionarial en la Administración Local. 


\section{b) Funcionarios de carrera. La Habilitación Nacional}

Dentro de este epígrafe se pueden situar dos tipos de funcionarios, los que comúnmente se conocen con el nombre de «propios» del Municipio, y los antiguos, pertenecientes a Cuerpos Nacionales y actualmente con «Habilitación Nacional».

Respecto a los funcionarios con Habilitación Nacional, los aspectos más importantes que contempla la Ley de Bases son los siguientes:

- Son funcionarios de carrera con Habilitación Nacional los sometidos al Estatuto Funcionarial.

- Su ejercicio supone tener «autoridad».

- Funciones públicas reservadas a este personal: fe pública y asesoramiento legal preceptivo, control y fiscalización interna de la gestión económico-financiera y presupuestaria, funciones contables de tesorería y recaudación.

- Posible incompatibilidad de la Ley de Bases con la filosofía de la Ley de Medidas, ésta potencia el puesto de trabajo frente a la Ley de Bases, que sin citar a los Cuerpos, tiene cierto contenido corporativo, pues se describen y definen funciones, lo que equivale a reconocer la existencia de Cuerpos, aunque no se consagran como tales.

- Por atentar contra la autonomía municipal, la Ley de Bases ha sido recurrida ante el Tribunal Constitucional por las Comunidades Autónomas de Galicia y Cataluña, recurso aún no resulto, al invadir, según dichas Comunidades Autónomas, la esfera de sus competencias por el Estado, estableciendo las bases de la convocatoria, la selección de este personal a nivel nacional, etcétera.

- El legislador de la Ley de Bases ha seguido el criterio de la jurisprudencia del Tribunal Constitucional (Sentencia de 7 de abril de 1983), donde se declaraba constitucional la existencia de Cuerpos Nacionales de Administración Local, hoy de Habilitación Nacional, y su selección de ámbito nacional, con arreglo a unos criterios básicos.

- En cuanto al tema de la movilidad funcionarial, el intercambio de funcionarios locales típicos de cada organización, según el artículo 101 de la Ley de Bases, los puestos de trabajo de funcionarios en las Corporaciones Locales se proveen por concurso de méritos entre funcionarios de cualquiera 
de las Administraciones públicas. Sin embargo, el artículo 17 básico y estatutario de la Ley de Medidas, en su primer punto establece la intercambiabilidad absoluta de cualquier funcionario perteneciente a cualquier Administración, en virtud de la mejor utilización de los recursos, pero en el punto 2 restringe el acceso de los funcionarios locales a la Administración del Estado y sólo pueden ir a la Administración de las Comunidades Autónomas.

c) Contratados en régimen de derecho laboral

- Se hace mediante oferta de empleo público.

- De acuerdo con los principios de igualdad, mérito y capacidad, con publicidad (art. 91.2 de la LB).

- Se reitera la igualdad de oportunidades (art. 103 de la LB).

- El personal laboral lo selecciona la Corporación Local (art. 103 de la LB).

- El sistema de selección es el tradicional (concurso, oposición, concurso-oposición libre), según la Ley de Bases (art. 91.2).

d) Personal eventual que desempeña puestos de confianza o asesoramiento especial

Las características de este personal son las siguientes:

- No son las del artículo 5.1 del Estatuto de la Función Pública de 1963, Ley Articulada de Funcionarios, que entonces era personal de confianza o de asesoramiento especial, en puestos de trabajo no reservados a funcionarios de carrera, pues este artículo ha sido derogado expresamente por la Ley de Medidas.

- Cesan al producirse el cese de la autoridad a la que prestan su función de confianza o asesoramiento.

- Por sus características, su nombramiento y cese es libre; éste se atribuye al Presidente de la Corporación Local correspondiente.

- Su número, características y retribuciones se establecen previamente, y la modificación de estas especificaciones sólo se puede hacer con motivo del Presupuesto anual de cada Corporación. 
5. INTENTO DE UNA SÍNTESIS A TRAVÉS DE DOS ESTATUTOS DE FUNCIONARIOS: EL ESTATUTO GENERAL DE LA FUNCIÓN PÚBLICA Y EL ESTATUTO PARTICULAR DE LA LOCAL

Es un tema arduo, complejo y además responde a un mandato legal, en este caso el de la Disposición Final Primera, apartado c) de la Ley de Bases, que prescribe un plazo de un año, en el que hay que refundir disposiciones muy dispares entre ellas, el Reglamento de Funcionarios Locales de 1952 , si bien se dice ha de hacerse de acuerdo con criterios armonizadores y con claridad.

a) El Título VII de la Ley Reguladora de las Bases del Régimen Local

El examen de este Título de la Ley de Bases nos impone determinadas precisiones:

- Si es un auténtico Estatuto de Funcionarios Locales.

- Es posterior, en el tiempo, a la Ley de Medidas.

- En cuanto a su prevalencia, plantea cuestiones, como la de si tiene rango suficiente para derogar, en su caso, preceptos de la Ley de Medidas.

- Técnicamente ha sido calificado de más perfecto que la regulación funcionarial de la Ley de Medidas.

- Recurrido, como se ha dicho, por inconstitucionalidad.

- Contiene disposiciones relativas a la generalidad del personal de las Entidades Locales, comunes a los funcionarios de carrera, a la selección y formación de los funcionarios con habilitación de carácter nacional y al sistema de provisión de plazas, a la selección de los restantes funcionarios y reglas sobre provisión de puestos de trabajo. Finaliza con normas relativas al personal laboral y eventual.

b) El Reglamento de Funcionarios de Administración Local, aprobado por Decreto de 30 de mayo de 1952

No es momento de juicio sobre su naturaleza, características, alcance y significado, pero se ha de refundir con los demás Reglamentos de la antigua Ley, hoy derogada, de Régimen Local, en un solo cuerpo legal, actualizándose y acomodándose con las demás disposiciones reglamentarias. 
La clasificación funcionarial del Reglamento de Funcionarios Locales es muy pormenorizada, en su momento avanzada, y responde al siguientes esquema (13).

- Funcionarios (art. 1):

a) por la permanencia (art. 2)

- en propiedad

- personal temporal: interino accidental

b) por la naturaleza de sus funciones

1. Cuerpos Nacionales: Secretarios

Interventores

Depositarios

Directores.de Bandas de Música

Civiles (Cuerpo hoy desaparecido por la Ley de Bases).

2. Que no constituyen Cuerpos Nacionales:

Administrativos (arts. 227 a 239)

Técnico-administrativos:

- Oficial Mayor

- Jefe de Sección

- Jefe de Negociado

- Oficiales

Auxiliares

3. Técnicos (arts. 240 a 248):

Superiores

Auxiliares

Asimilados

4. De servicios especiales (arts. 249 a 258)

5. Subalternos (arts. 259 a 261)

6. Obreros de plantilla (art. 5)

(13) Vid. Mahillo Santos, Juan: La Función Pública Local españota y sus proble mas actuales, Instituto de Estudios de Administración Local, Madrid, 1976, 550 págs. en cuarto, obra descriptiva que recoge la tesis de Diplomado en Administracion Local de su autor, donde nos ofrece un panorama bastante completo de la Función Pública Local en la època; es decir, en el periodo inmediatamente anterior al actual.

En este apartado las alusiones de articulos que se hacen entre parentesis son referencias al Reglamento de Funcionarios de Administración Local, aprobado por Decreto de 30 de mayo de 1952. 
- No funcionarios:

a) Por transitoriedad de los trabajos (art. 6)

- Temporeros (funciones administrativas)

- Eventuales (funciones no administrativas)

b) Por la forma de gestión de los servicios públicos, susceptibles de tener un órgano especial o en forma de empresa privada o mixta.

- Contratados (de dedicación no periódica ni permanente) (art. 8):

- Con relación laboral (funciones de índole manual)

- Con relación administrativa (las demás funciones) (hoy prohibida la contratación administrativa de este personal, según la Ley de Medidas).

Se hace esta descripción para conocer la situación, hasta la fecha, de donde ha de partir la reforma por aplicación de un régimen normativo ambivalente, al que se ha hecho referencia (14).

c) Mosaico de soluciones y situaciones en el ordenamiento jurídico funcionarial local

Se evidencia la situación pulverizada por las siguientes circunstancias:

- Desde las denominaciones hasta las situaciones, definiciones y complejidades.

(14) Vid. ob. cit., Mahillo Santos, Juan: La Función Pública Local española..., que al hacer una clasificación del personal de plantilla ofrece la siguiente panoramica en la p. 108 :

Cuerpos Nacionales

Administrativo ... $\left\{\begin{array}{l}\text { Técnicos-admvos. } \\ \begin{array}{l}\text { Auxiliares } \\ \text { Otros }\end{array}\end{array}\right.$

\section{Secretarios}

Interventores

Depositarios

Directores de Banda

Oficial mayor

Viceinterventor

Jefes de Seccion

Subjefes de Sección

Jefes de Negociado

Subjefes de Negociado

Oficiales 
- Funcionario es, en definitiva, aquel que hace de su vida profesión.

- Pluralidad y variedad en el ámbito local, de funciones, situaciones, atipicidades, singularidades, en definitiva, complejidad.

- Hay una normativa prolija, compleja, diferente.

- Más de 8.000 organizaciones, con peculiaridades en cada una, acentuadas por la autonomía local.

- Es difícil una reglamentación orgánica común, se puede producir un vacío normativo, sobre todo en la mayor parte de los Municipios de escasa entidad, al intentar la aplicación de la reforma, que lo es para todos.

\section{LA REFORMA BUROCRATICA ESPAÑOLA, ACONTECIMIENTO IMPORTANTE}

En primer lugar, explicar el porqué de estas reflexiones, en ocasiones enunciados, en oiras ligeras explicitaciones $y$, en general, sugerencias.

Se ha intentado, simplemente, exponer la situación de hoy, en

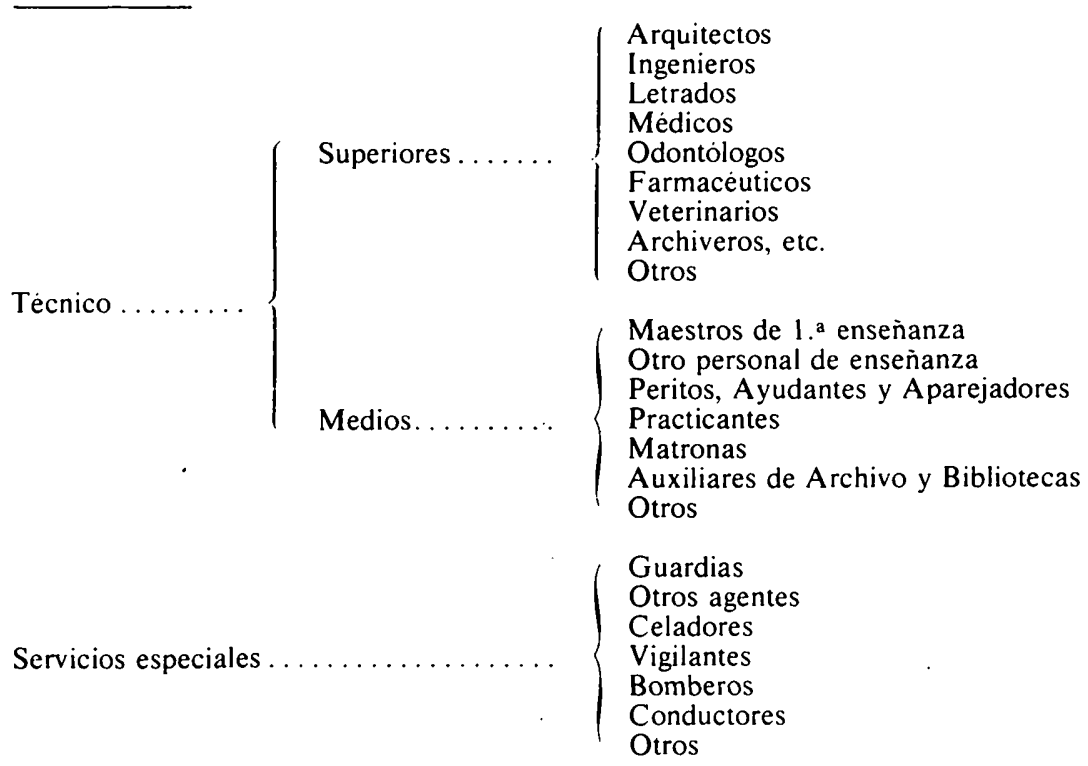

Subalternos. 
evidencia en relación con la Administración Local de nuestros días.

Se demuestra de forma palmaria la necesidad de concretar un Estatuto del funcionario público español, situación agravada en la esfera local; de ahí el eco a nivel de diario, revista, publicación periódica, de una u otra naturaleza (15).

Existen evidentes problemas, como es una tradicional separación entre la función pública local y la del Estadó. En la actualidad este planteamiento se complica, al establecerse como intermedia y existir una Administración nueva: la autonómica. Son tres círculos concéntricos, sin tangencias pero con trasvases, como se ha dicho, y es necesario un tratamiento común, iniciado ya por la Ley de Medidas, intento de un Estatuto parcial, aplicado parcialmente, al declararse más del 50 por ciento de sus Medidas, bases del régimen estatutario de los funcionarios públicos, aplicables al personal de todas las Administraciones públicas. La cuestión radica en si por esta vía se va a conseguir la ideal y necesaria unificación, es decir

(15) Sirvan de ejemplo las siguientes referencias y autores, alfabéticamente ordenadas:

- Barranco, Juan: "Sobre el Proyecto de Ley de Bases de Régimen Local", Diario El Pais, 7 noviembre 1983.

- Benitez de LUGo, José Mariano: Entrevista en la Revista Cunal, núm. 459, marzo 1983, pp. 209-213.

- Corella Monedero, José Mario: «Los Cuerpos Nacionales de Administración Local", Diario $A B C, 17$ junio 1984.

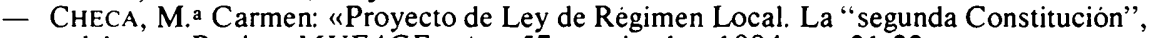
a debate». Revista $M U F A C E$, num. 57, septiembre 1984, pp. 21-22.

- De la Quadra-Salcedo, Tomás: Entrevista en la Revista Certamen, núm. 492, marzo 1984, pp. 109-112.

- Diez Del RonCAL, Angel: "El personal al servicio de las Entidades Locales", El Socialista, núm. 372, 1 enero 1985.

- Garcia de la Riva, Andrés: (Subsecretario del Ministerio de Administración Territorial) Entrevista en la Revista Certamen, num. 480, febrero 1983, pp. 79-82.

- Gonzalez-Berenguer, Jose Luis: "Sobre los Cuerpos Nacionales de Administración Local", Revista Municipalia, num. 346, diciembre 1983, pp. 7-21.

- GONZALEZ-HABa GuISADO. Vicente M.a: "El reto del nuevo Regimen Local», Diario El Pais, 12 julio 1984.

- Gonzalez-Haba Guisado, Vicente M.a: "Rasgos de la Función Pública Local», Revista Certamen, num. 501, enero 1985, pp. 7-9.

- Lobato BRIMe, Francisco: "La Funcion Pública Local en la Ley de Medidas para la reforma de la Función Pública", Revista Municipalia, num. 355, octubre 1984, pp. 13-19.

- Millana DE YNES, José Cruz: "La Función Pública Local en el actual Proyecto de Ley reguladora de las bases del Regimen Local», Revista Municipalia, núm. 352, julio 1984, pp. 17-26.

- PRATS. Joan: «Administración publica y transicion democratica», Revista Pensamiento Iberoamericano. núm. $5 \mathrm{~b}$, pp. 445-462.

- SOSA WAGNER. Francisco: Entrevista en la Revista El Funcionario Municipal, num. 279, diciembre 1983, pp. 437-440.

- Sosa Wagner, Francisco: "La Función Pública Local», Diario $A B C, 2$ julio 1984. 
ausencia de diferencias y trato entre el personal al servicio de las distintas Administraciones y, en concreto, de los tres sectores enunciados.

El momento actual supone un paréntesis abierto a una reforma, ambiciosa por ser total; con riesgos, pues existen intereses; con susceptibilidades, ya que el tema es muy sensible con posibles incidencias de todo tipo, incluso político; con participaciones deseables, teniendo en cuenta derechos mayoritaria o minoritariamente compartidos; sin intolerancias, eludiendo posturas arrogantes en beneficio, no sólo de los servidores públicos, sino de la propia sociedad española.

El momento, por el contenido de la reforma, la estrategia de la misma, la carga tradicional, la adecuación a nuevas estructuras, es polémico. Potenciada la discusión por una coyuntura desfavorable, el desempleo y paro, el juvenil en especial; con otro componente importante, radical cambio en la estructura del empleo en la Administración pública; con un rejuvenecimiento acusado, proceso acelerado por medidas como la anticipación de la jubilación y otras posibles de futuro.

Otra circunstancia de alteración de los parámetros tradicionales en la consideración del tema, es que la Administración se ha convertido en el primer empleador de mano de obra, de alguna forma cualificada. Antes compartía este papel con el sector privado, ahora es el principal protagonista y se convierte en empresa de empleo, en un principio segura, frente al riesgo de la privada, a causa de la crisis económica, siempre con la amenaza de la posible quiebra, que arrastra a sus servidores.

Otro hecho evidente es la laborización de la función pública; cada vez se tiende más a no distinguir, como un administrado especial al funcionario $y$ en equiparación, tratarle como trabajador al servicio de la Administración. Se da, sobre todo en la Administración Local, la circunstancia de clasificar un mismo puesto de trabajo de forma distinta, pues corresponde a cada Corporación Local aprobar anualmente su plantilla, que comprende todos los puestos de trabajo, tanto los reservados a funcionarios como a personal laboral, o en su caso eventual. Sólo se produce un supuesto de reserva legal, de determinadas funciones públicas, que se hace en favor de funcionarios con carácter de habilitación nacio- 
nal (16), si bien, incluso en alguno de estos supuestos de funciones, las relativas al ejercicio de las mismas en el área económico-financiera, excepcionalmente, pueden ser atribuidas a miembros de la Corporación, o funcionarios sin habilitación nacional.

Cuestión también importante es la situación actual de la función pública, no sólo en España, que se encuentra en un momento de crisis, ante la que se debe reaccionar congruentemente y una de las formas de esta reacción es la de ofrecer un campo jurídico, un estatuto, una normativa, coherente y suficiente, que por sí sola no es bastante, aunque supone un primer paso para superar la no deseada situación en que nos encontramos.

(16) El articulo 92.2 de la Ley Reguladora de las Bases del Régimen Local, Ley $7 / 1985$, de 2 de abril, determina cuáles son las funciones públicas cuyo cumplimiento queda reservado con exclusividad a un personal, funcionarios de carrera, que está sujeto al Estatuto funcionarial, las caracteristicas de estas funciones, la una generica: que impliquen ejercicio de autoridad, y la otra especifica, sectorializada por actividades, como es la fe pública, el asesoramiento legal preceptivo, control y fiscalizacion interna de la gestion económico' financiera y presupuestaria, incluyendose las tareas de contabilidad y tesoreria.

Pero no solamente son estas funciones públicas, por supuesto. las más importantes, sino aquellas otras que en desarrollo de la Ley se reserven a funcionarios, léase con habilitacion nacional, pues el punto 3 de este articulo 92 declara necesarias las funciones de secretaria y de control y fiscalización interna de la gestion economico-financiera. Pero, además, se reservan a funcionarios con habilitacion nacional dichas funciones y sólo en el caso de determinadas actividades económico-financieras por el numero 4 del referido articulo 92 , excepcionalmente, la responsabilidad administrativa de la contabilidad, tesoreria y recaudacion, se puede atribuir a miembros de la Corporacion o funcionarios sin habilitacion nacional.

Pero esta salvedad que acabamos de citar es limitada, pues, además de su carácter de excepción, no se refiere a todas las funciones publicas, necesarias en todas las Corporaciones, sólo a los aspectos contables y de tesoreria y recaudación, por supuesto, no a la fe pública y al asesoramiento legal preceptivo, como tampoco al control y fiscalizacion interna de la gestión económico-financiera y presupuestaria.

Delimitada de esta manera la reserva de actividades, un analisis superficial demuestra que, aunque no existan Cuerpos Nacionales, sino funcionarios con habilitacion nacional, en la actualidad lo que si se ha hecho es definir la actividad de los Cuerpos; si subjetivamente no tienen razón de ser, objetivamente, sin embargo, están descritos. Pero, además, las funciones que se les atribuyen son las más importantes, necesarias. reservadas, todo ello configura una situación de importancia y trascendencia que se justifica con garantias tan fundamentales como las de la objetividad, imparcialidad e independencia en el ejercicio de. la funcion (vid. articulo 92.2 in fine).

Por todo lo expuesto, la Ley Reguladora de las Bases del Régimen Local supone un reducto de funcionarizacion frente a la posible laborizacion de la funcion publica, y en otros terminos se enfrenta de alguna manera con la filosofia insita en la Ley de Medidas para la Reforma de la Funcion Pública. claramente anticorporativista. no solo por su articulo 19.2. relativo a la selección y a la formación de los Tribunales de esta naturaleza. sino por su espiritu y carácter, del que de alguna manera es una manifestación su disposición adicional novena, sobre los Cuerpos de funcionarios existentes. 


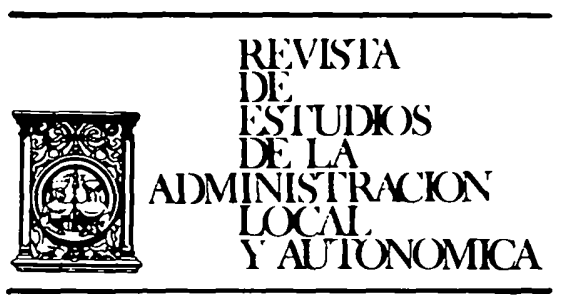

\section{CRONICAS}


REALA-1986, núm. 230. CARRASCO CANALS, CARLOS. ESPECIAL INCIDENCIA DEL ACTUAL RE...

REALA-1986, núm. 230. CARRASCO CANALS, CARLOS. ESPECIAL INCIDENCIA DEL ACTUAL RE... 